\title{
Low risk of lymph node metastasis in 495 early gastric cardiac carcinomas: a multicenter clinicopathologic study of 2101 radical gastrectomies for early gastric carcinoma
}

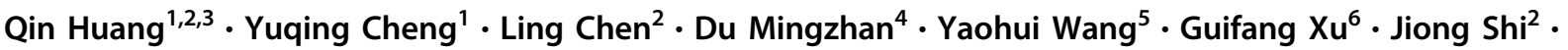 \\ Xiangshan Fan ${ }^{2} \cdot$ Xiaoli Zhou $^{1} \cdot$ Yifeng Zhang $^{5} \cdot$ Linchuan Guo $^{4} \cdot$ Tianyun Liu $^{2} \cdot$ Dan Zhou ${ }^{7} \cdot$ Hiroshi Mashimo $^{8}$. \\ Jason S. Gold $\mathbb{D}^{9} \cdot$ Xiaoping Zou ${ }^{6}$ and The Jiangsu Province Early Gastric Carcinoma Multicenter Study Team
}

Received: 11 February 2018 / Revised: 26 March 2018 / Accepted: 29 March 2018 / Published online: 25 May 2018

(c) United States \& Canadian Academy of Pathology 2018

\begin{abstract}
Clinical decision-making on endoscopic vs. surgical resection of early gastric cardiac carcinoma remains challenging because of uncertainty on risk of lymph node metastasis. The aim of this multicenter study was to investigate risk factors of lymph node metastasis in early gastric cardiac carcinoma. Guided with the World Health Organization diagnostic criteria, we studied 2101 radical resections of early gastric carcinoma for risk factors associated with lymph node metastasis, including tumor location, gross pattern, size, histology type, differentiation, invasion depth, lymphovascular, and perineural invasion. We found that the risk of lymph node metastasis was significantly lower in early gastric cardiac carcinomas $(6.7 \%, 33 / 495)$, compared with early gastric non-cardiac carcinomas $(17.1 \%, 275 / 1606)(p<0.0001)$. In early gastric cardiac carcinoma, no lymph node metastasis was identified in intramucosal carcinoma (0/193) and uncommon types of carcinomas $(0 / 24)$, irrespective of the gross pattern, size, histologic type, differentiation, and invasion depth. Ulceration, size $>3 \mathrm{~cm}$, and submucosal invasion were not significant independent risk factors for lymph node metastasis. In 33 early gastric cardiac carcinomas with lymph node metastasis, either lymphovascular invasion or poor differentiation was present in $16(48.5 \%)$ cases and together in six cases. By multivariate analysis, independent risk factors of lymph node metastasis in early gastric cardiac carcinoma included lymphovascular invasion (Odds Ratio (OR): 7.6, 95\% Confidence Interval (CI): 2.8-20.2) $(p<$ $0.0001)$ and poor differentiation (OR: 6.0, 95\% CI: 1.4-25.9) $(p<0.05)$. In conclusion, lymph node metastasis was not identified in early gastric cardiac intramucosal carcinoma and uncommon types of carcinoma. The risk of lymph node metastasis was also significantly lower in tumors with submucosal invasion, especially for cases without lymphovascular invasion or poor differentiation. These results lend support to the role of endoscopic therapy in the treatment of patients with early gastric cardiac carcinoma.
\end{abstract}

These authors contributed equally: Qin Huang, Yuqing Cheng, Ling Chen, Mingzhan Du, Yaohui Wang.

Qin Huang

qinhuang0122@gmail.com

1 Department of Pathology, the Changzhou Second Hospital, Changzhou, China

2 Departments of Pathology, the Nanjing Drum Tower Hospital, Nanjing, China

3 Pathology and Laboratory Medicine, the Boston VA Healthcare System and Harvard Medical School, West Roxbury, MA, USA

4 Department of Pathology, the Soochow University First Hospital, Suzhou, China

\section{Introduction}

Gastric cardiac carcinoma arises in the gastric cardia below the gastroesophageal junction [1-3]. Because of the paucity

5 Department of Pathology, the Jiangsu Provincial Hospital of Traditional Chinese Medicine, Nanjing, China

6 Departments of Gastroenterology, the Nanjing Drum Tower Hospital, Nanjing, China

7 Quality Healthcare Consultant, Lexington, MA, USA

8 Departments of Gastroenterology, the Boston VA Healthcare System and Harvard Medical School, West Roxbury, MA, USA

9 Departments of Surgery, the Boston VA Healthcare System and Harvard Medical School, West Roxbury, MA, USA 
of clinical symptoms, most patients with tumors diagnosed at advanced stages have a poor prognosis. The 5-year survival rate was reported to be lower than $10 \%$ [1]. At present, the best strategy proven to improve patient survival is early diagnosis and resection as gastric cardiac carcinomas at early (pT1) stage have significantly improved 5-year survival rates of over $90 \%$ [2]. With advances in endoscopic therapy, early gastric carcinoma can be effectively resected by endoscopic mucosal resection or endoscopic submucosal dissection with fewer complications, better preservation of gastric function, and lower cost compared with surgical resection [4-6]. However, the clinical decision making for choosing endoscopic vs. surgical resection for early gastric carcinoma is based on the clinicopathologic finding of negligible risk of lymph node metastasis. Unfortunately, risk factors for nodal metastasis in early gastric cardiac carcinoma remain elusive.

Gastric cardiac carcinoma is uncommon in Western countries as well as some East Asian countries such as Japan and Korea [7]. There are a handful of single-center studies with small sample sizes that show a lower risk of lymph node metastasis in early gastric cardiac carcinoma [8-10] compared with early gastric non-cardiac carcinoma [2, 10-12]. These limited single-center experiences on assessment of risk of lymph node metastasis in early gastric cardiac carcinoma require in-depth investigation with large samples. Herein, we conducted a multicenter clinicopathologic study of risk factors associated with lymph node metastasis in 495 consecutive early gastric cardiac carcinoma radical resections in the Jiangsu Province in China where gastric cancer remains one of leading malignancies in terms of incidence and mortality. Notably, the most important confounding factors for investigation of early gastric cardiac carcinoma, such as Barrett's esophagus and esophageal adenocarcinoma are rare in this study patient population [13-16].

\section{Materials and methods}

\section{Study design, patient selection, and groups}

In this multicenter retrospective study, we searched the electronic pathology database stored in the Departments of Pathology of four participating tertiary medical centers for the final pathologic diagnosis of gastric cancer in radical gastrectomy specimens over the 11-year period from 2005 through 2016 for The Nanjing Drum Tower Hospital, from 2011 to 2015 for The Jiangsu Provincial Hospital of Traditional Chinese Medicine, and from 2011 to 2016 for The Changzhou Second Hospital and The Affiliated First Hospital of Soochow University. Each pathology report was scrutinized to exclude the cases with tumor invasion into the muscularis propria and beyond. The resection cases with tumor invasion limited to the mucosa and submucosa (pT1) were selected as early gastric carcinoma for the study. All histology slides, including special stains, of each case were retrieved and investigated. Excluded were esophageal adenocarcinoma, stump carcinoma, lymphoma, synchronous carcinomas, high-grade dysplasia, tumor invasion into the muscularis propria, and cases with a history of neoadjuvant therapy before radical resection. In addition, cases without histology slides or paraffin blocks for recuts were also eliminated. Pathology reports of all selected cases were reviewed for patient demographic information, tumor epicenter location, size, shape, surface characteristics, and relationship to the gastroesophageal junction. In cases with equivocal information on tumor location, size, and shape, the original upper endoscopic reports and images along with the surgical resection notes were reviewed for accurate information. All identifying patient information was deleted from the data sheet to protect patient privacy. The pathology accession number of each case was used as the reference for all communication and data analyses. The study protocol was approved by the Medical Ethics Committee of all 4 participating medical centers.

\section{Pathologic study}

All study pathologists had over 5-year clinical practice experience with strong interest in gastrointestinal pathology and applied the World Health Organization criteria to guide early gastric carcinoma diagnosis in daily practice [17]. Early gastric cardiac carcinoma was defined as tumors with epicenters located in a narrow region of about $3 \mathrm{~cm}$ below the gastroesophageal junction line, as previously described [2, 3]. Early gastric non-cardiac carcinoma referred to early gastric carcinomas with epicenters in other regions of the stomach. Tumor gross characteristics were sub-grouped into five patterns as follows: (1) polypoid/protruding (type 0-I), (2) slightly elevated with rough surface (type 0-IIa), (3) flat (type 0-IIb), (4) slightly depressed with erosion (type 0-IIc), and (5) excavated with ulcer involving the muscularis mucosae and beyond (type 0-III) [17]. Tumor size was measured after routine overnight formalin fixation. Depth of tumor invasion was determined microscopically from the muscularis mucosae to the lowest point of invasion and divided into four categories: (1) M2, tumors limited to the lamina propria without the involvement of the muscularis mucosae; (2) M3, tumors involving the muscularis mucosae; (3) SM1, tumor invasion into the superficial submucosa $(<0.5 \mathrm{~mm}$ from the muscularis mucosae); and (4) SM2, tumor involvement of the deep submucosa ( $>0.5 \mathrm{~mm}$ from the muscularis mucosae) [2]. With the World Health Organization criteria on gastric carcinoma classification and differentiation [17], gastric adenocarcinoma was divided into five main types: tubular, papillary, mucinous, poorly cohesive, and mixed. Also tabulated and analyzed were other rare variants such as adenosquamous and neuroendocrine carcinomas, carcinoma with 
lymphoid stroma, mixed mucinous and tubular/papillary adenocarcinoma, and mixed poorly cohesive carcinoma with tubular/papillary adenocarcinoma.

Appropriate immunohistochemical and in situ hybridization tests were employed to confirm the diagnosis of neuroendocrine carcinoma and carcinoma with lymphoid stroma, respectively, on the basis of the initial impression on routine hematoxylin-eosin stained tumor sections [2]. The World Health Organization tumor differentiation grading system on gastric carcinoma was adopted and applied primarily to tubular and papillary adenocarcinomas, in which well differentiated tumors were defined as having wellformed neoplastic glands in over $95 \%$ of the estimated tumor volume, while poorly differentiated tumors were defined as having well-formed glands in $<49 \%$ of the tumor, and thus moderately differentiated tumors were defined as having a proportion of discernable glands that was in between [17]. Poorly cohesive carcinoma, including signet-ring cell carcinoma, was thus classified as poorly differentiated. In each case, lymphovascular invasion, and perineural invasion were also recorded and analyzed. The tumor $\mathrm{pN}$ staging was carried out, according to the American Joint Committee on Cancer staging manual 7th edition [12].

As a routine quality control procedure for this four-center study, cases with equivocal diagnoses were discussed between the study pathologists via teleconferences. The senior study pathologist and organizer $(\mathrm{QH})$ often traveled to each center to help out, periodically audited the preliminary study results, and sometimes requested further investigations of unusual findings reported by study pathologists. Frequent discussions via emails or WeChat ${ }^{\mathrm{TM}}$ among study pathologists were regularly conducted to ensure a smooth study process.

\section{Statistical analysis}

Differences between the groups with and without lymph node metastasis were statistically analyzed and compared with respect to patient age, gender, tumor gross pattern, size, invasion depth, histology type, differentiation, lymphovascular, and perineural invasion. The $\chi^{2}$, Fisher's exact, or Kruskal-Wallis $\mathrm{H}$ test was utilized, where appropriate. A logistic regression analysis model was used for univariate and multivariate analyses on risk factors of lymph node metastasis. Statistical Package for Social Sciences (SPSS, version 17, Chicago, USA) was employed for all statistical analyses. $P$ values $<0.05$ were defined as statistically significant.

\section{Results}

Over the study period, a total of 12,050 radical gastrectomies for gastric cancer were reviewed. After exclusion of advanced gastric cancer $(N=9737)$ and disqualified $(N$ $=281$ ) cases, 2101 consecutive early gastric carcinoma cases were eligible for the study, in which 495 were classified as early gastric cardiac carcinoma $(23.6 \%, 259 / 2101)$ and $1606(76.4 \%, 1606 / 2101)$ were as early gastric noncardiac carcinoma.

\section{Lymph node metastasis}

The average number of lymph nodes retrieved in each case was 17.2 (range: 2-61) for the early gastric cardiac carcinoma group and 18.1 (range: $2-65$ ) for the early gastric noncardiac carcinoma group. Lymph node metastasis was identified in $308(14.7 \%$, 308/2101) cases and was significantly more frequent in early gastric non-cardiac carcinomas $(17.1 \%, 275 / 1606)$ than in early gastric cardiac carcinomas $(6.7 \%, 33 / 495)(p<0.0001)$. In 33 early gastric cardiac carcinoma cases with nodal metastasis, 26 (78.8\%, 26/33), $6(18.2 \%, 6 / 33)$, and $1(3 \%, 1 / 33)$ cases were staged as pN1, pN2, and pN3, respectively. As shown in Table 1, there were no significant differences in patient age, gender, and tumor macroscopic growth pattern between early gastric cardiac carcinomas with lymph node metastasis and early gastric cardiac carcinomas without. For tumors with a size of $0.9 \mathrm{~cm}$ or smaller, the risk of lymph node metastasis was significantly lower $(p<0.05)$. In contrast, for tumors measuring $3 \mathrm{~cm}$ or greater, the risk of lymph node metastasis was significantly higher $(p<0.0001)$. There was no significant difference in the risk of lymph node metastasis for tubular adenocarcinoma, papillary adenocarcinoma, mucinous adenocarcinomas, and poorly cohesive carcinoma. Although most early gastric cardiac carcinoma cases with lymph node metastasis $(78.8 \%, 26 / 33)$ were tubular $(54.5 \%$, $18 / 33)$ or papillary $(24.4 \%, 8 / 33)$ adenocarcinomas (Fig. 1), mixed poorly cohesive carcinoma with tubular/papillary adenocarcinomas $(18.2 \%, 6 / 33)$ were substantial. One case $(3.0 \%, 1 / 33)$ with lymph node metastasis was mucinous adenocarcinoma. No lymph node metastasis was found in uncommon types of early gastric cardiac carcinoma, such as neuroendocrine (Fig. 2) and adenosquamous (Fig. 3) carcinomas, carcinoma with lymphoid stroma (Fig. 4), and mixed mucinous and tubular/papillary adenocarcinomas. However, the risk of nodal metastasis was significantly increased in early gastric cardiac carcinomas with mixed poorly cohesive carcinoma and tubular/papillary adenocarcinomas $(p<0.05)$.

As shown in Table 1, well differentiated early gastric cardiac carcinomas exhibited a significantly lower risk of lymph node metastasis $(p<0.0001)$, whereas poorly differentiated early gastric cardiac carcinomas showed a significantly higher risk of lymph node metastasis $(p<0.0001)$. The risk of lymph node metastasis was also significantly increased in early gastric cardiac carcinomas with lymphovascular invasion $(p<0.00001)$, but not with perineural 
Table 1 Risk factors of lymph node metastasis in early (pT1) gastric cardiac carcinoma

\begin{tabular}{|c|c|c|c|c|c|}
\hline \multicolumn{2}{|l|}{ Clinicopathologic factor } & \multicolumn{3}{|c|}{ Lymph node metastasis $(\%)$} & \multirow[t]{3}{*}{$p$ value } \\
\hline & & \multirow{2}{*}{$\begin{array}{l}\text { Total number } \\
495\end{array}$} & \multirow{2}{*}{$\begin{array}{l}\text { Present } \\
33(6.7)\end{array}$} & \multirow{2}{*}{$\begin{array}{l}\text { Absent } \\
462(93.3)\end{array}$} & \\
\hline Number & & & & & \\
\hline \multirow[t]{3}{*}{ Gender } & Male & 374 (75.6) & $24(72.7)$ & $350(75.8)$ & NS \\
\hline & Female & $121(24.4)$ & $9(27.3)$ & $112(24.2)$ & \\
\hline & $\mathrm{M} / \mathrm{F}$ ratio & 3.1 & 2.7 & 3.1 & NS \\
\hline \multirow[t]{7}{*}{ Age (Year) } & Average (SD) & $65.4(8)$ & $64.5(9.5)$ & $65.4(7.9)$ & NS \\
\hline & $\leq 49$ & $17(3.4)$ & $1(3)$ & $16(3.5)$ & NS \\
\hline & $50-59$ & $88(17.8)$ & $9(27.3)$ & $79(17.1)$ & NS \\
\hline & $60-69$ & $231(46.7)$ & $13(39.4)$ & $218(47.2)$ & NS \\
\hline & $\geq 70$ & $159(32.1)$ & $10(30.3)$ & $149(32.3)$ & NS \\
\hline & $<60$ & $105(21.2)$ & $10(30.3)$ & $95(20.6)$ & NS \\
\hline & $\geq 60$ & $390(78.8)$ & $23(69.7)$ & 367 (79.4) & \\
\hline \multirow[t]{8}{*}{ Macroscopic feature } & $0-\mathrm{I}$ & $45(9.1)$ & $2(6.1)$ & $43(9.3)$ & NS \\
\hline & 0-IIa & $95(19.2)$ & $8(24.2)$ & $87(18.8)$ & NS \\
\hline & $0-\mathrm{IIb}$ & $136(27.5)$ & $8(24.2)$ & $128(27.7)$ & NS \\
\hline & 0-IIc & $138(27.9)$ & $10(30.3)$ & $128(27.7)$ & NS \\
\hline & 0-III & $81(16.4)$ & $5(15.2)$ & $76(16.5)$ & NS \\
\hline & $0-$ I to $0-$ IIa & $140(28.3)$ & $10(30.3)$ & $130(28.1)$ & NS \\
\hline & $0-\mathrm{IIb}$ & $136(27.5)$ & $8(24.2)$ & $128(27.7)$ & NS \\
\hline & $0-$ IIc $+0-$ III & $219(44.2)$ & $15(45.5)$ & $204(44.2)$ & NS \\
\hline \multirow[t]{4}{*}{ Size $(\mathrm{cm})$} & $\leq 0.9$ & $76(15.4)$ & $1(3)$ & $75(16.2)$ & $<0.05$ \\
\hline & $1.0-1.9$ & $159(32.1)$ & $7(21.2)$ & $152(32.9)$ & NS \\
\hline & $2.0-2.9$ & $159(32.1)$ & $9(27.3)$ & $150(32.5)$ & NS \\
\hline & $>3.0$ & $101(20.4)$ & $16(48.5)$ & $85(18.4)$ & $<0.0001$ \\
\hline \multirow[t]{4}{*}{ Invasion depth } & M2 & $63(12.7)$ & 0 & $63(13.6)$ & $<0.05$ \\
\hline & M3 & $130(26.3)$ & 0 & $130(28.1)$ & $<0.0001$ \\
\hline & SM1 & $115(23.2)$ & $10(30.3)$ & $105(22.7)$ & NS \\
\hline & SM2 & $187(37.8)$ & $23(69.7)$ & $164(35.5)$ & $<0.0001$ \\
\hline \multirow[t]{8}{*}{ Histology type } & Tubular adenocarcinoma & $326(65.9)$ & $18(54.5)$ & $308(66.7)$ & NS \\
\hline & Papillary adenocarcinoma & $106(21.4)$ & $8(24.2)$ & $98(21.2)$ & NS \\
\hline & Poorly cohesive carcinoma & $11(2.2)$ & $2(6.1)$ & $9(1.9)$ & NS \\
\hline & Mucinous carcinoma & $7(1.4)$ & $1(3)$ & $6(1.3)$ & NS \\
\hline & Micropapillary adenocarcinoma ${ }^{a}$ & $11(2.2)$ & $1(3)$ & $10(2.2)$ & NS \\
\hline & Mixed PCC and tubular/papillary adenocarcinoma & $21(4.2)$ & $4(12.1)$ & $17(3.7)$ & $<0.05$ \\
\hline & Mixed mucinous and tubular/papillary adenocarcinoma & $14(2.8)$ & 0 & $14(3)$ & NS \\
\hline & $\begin{array}{l}\text { Neuroendocrine }(N=4) \text { medullary }(N=4) \text {, adenosquamous ( } N \\
=2 \text { ) carcinoma }\end{array}$ & $10(2)$ & 0 & $10(2.2)$ & NS \\
\hline \multirow[t]{3}{*}{ Differentiation } & Well & $158(31.9)$ & $2(6.1)$ & $156(33.8)$ & $<0.0001$ \\
\hline & Moderate & $226(45.7)$ & $15(45.5)$ & $211(45.7)$ & NS \\
\hline & Poorly & $111(24.4)$ & $16(48.5)$ & $95(20.6)$ & $<0.0001$ \\
\hline \multirow[t]{2}{*}{ Lymphovascular invasion } & Absence & $440(88.9)$ & $17(51.5)$ & 423 (91.6) & $<0.00001$ \\
\hline & Presence & $55(11.1)$ & $16(48.5)$ & $39(8.4)$ & \\
\hline \multirow[t]{2}{*}{ Perineural invasion } & Absence & 483 (97.6) & $31(93.9)$ & $452(97.8)$ & NS \\
\hline & Presence & $12(2.4)$ & $2(6.1)$ & $10(2.2)$ & \\
\hline
\end{tabular}

${ }^{a}$ Micropapillary adenocarcinoma was present as a minor component and excluded from the frequency (\%) calculation as a major subtype of carcinoma in the cohort 

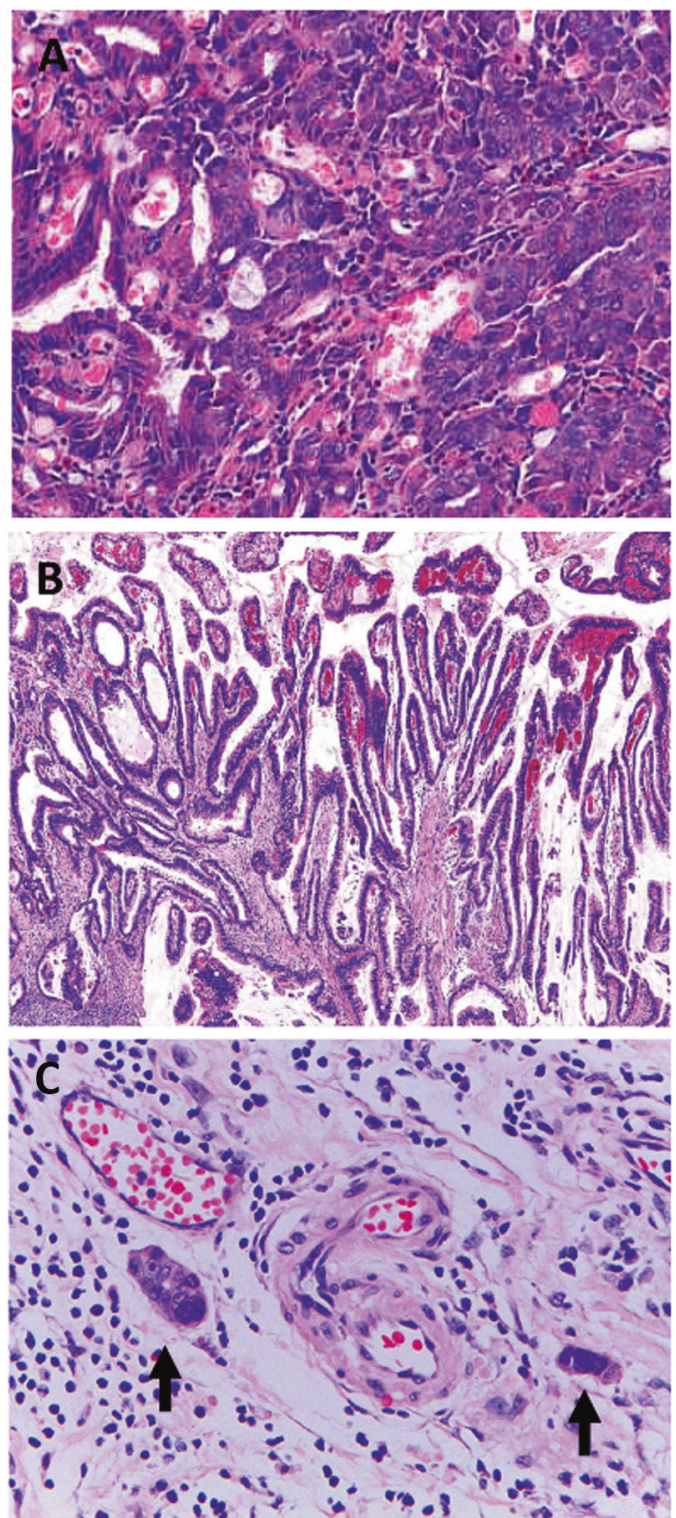

Fig. 1 Microscopic features of early tubular and papillary cardiac adenocarcinomas. a A poorly differentiated tubular adenocarcinoma exhibited solid and cribriform growth patterns. b The tumor showed papillary morphology with well-defined fibrovascular cores lined by dysplastic columnar cells. c lymphovascular invasion (black arrows) was identified in the submucosa

invasion. By a multivariate logistic regression analysis (Table 2), poor differentiation and lymphovascular invasion were identified as significant independent risk factors of lymph node metastasis in early gastric cardiac carcinomas with odds ratios of $6.0 \quad(95 \%$ confidence interval: $1.41-25.88, p<0.05)$ and 7.6 (95\% confidence interval: $2.75-20.24, p<0.0001)$, respectively. Among 33 early gastric cardiac carcinoma cases with nodal metastasis, 16 $(48.5 \%, 16 / 33)$ showed lymphovascular invasion, 16 $(48.5 \%, 16 / 33)$ revealed poor differentiation, $6(18.2 \%, 5 /$ 33) exhibited both lymphovascular invasion and poor
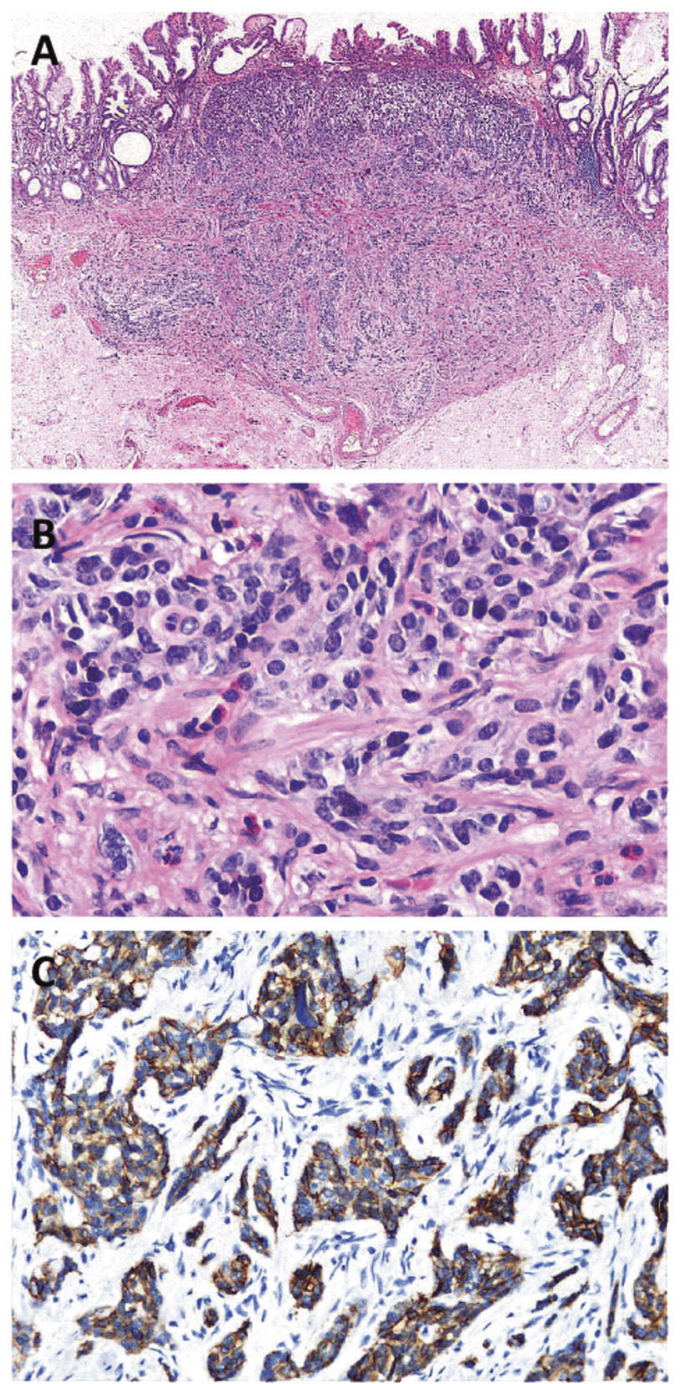

Fig. 2 Early gastric cardiac neuroendocrine carcinoma showing submucosal invasion with a sharp invasion front in the submucosal space (a) and demonstrating salt-pepper nuclear chromatin features (b) and immunoreactivity to synaptophysin (c)

differentiation. Only $7(21.2 \%, 7 / 33)$ cases did not demonstrate either. Thus, most early gastric cardiac carcinoma cases with nodal metastasis $(78.8 \%, 26 / 33)$ displayed either lymphovascular invasion or poor differentiation or both.

\section{Depth of invasion and lymph node metastasis}

In this cohort, the risk of lymph node metastasis was $6.3 \%$ (62/982) for intramucosal carcinoma and significantly increased to $22.0 \%$ (246/1119) for submucosal carcinoma ( $p$ $<0.0001$ ). As shown in Table 3, no lymph node metastasis occurred in intramucosal (M2 and M3 invasion depths) early gastric cardiac carcinomas. Despite more frequent submucosal invasion of early gastric cardiac carcinomas into SM1 (23.2\%) and SM2 (37.8\%), compared with early gastric noncardiac carcinomas $(19.7 \%$ and $31.2 \%$, respectively), the 


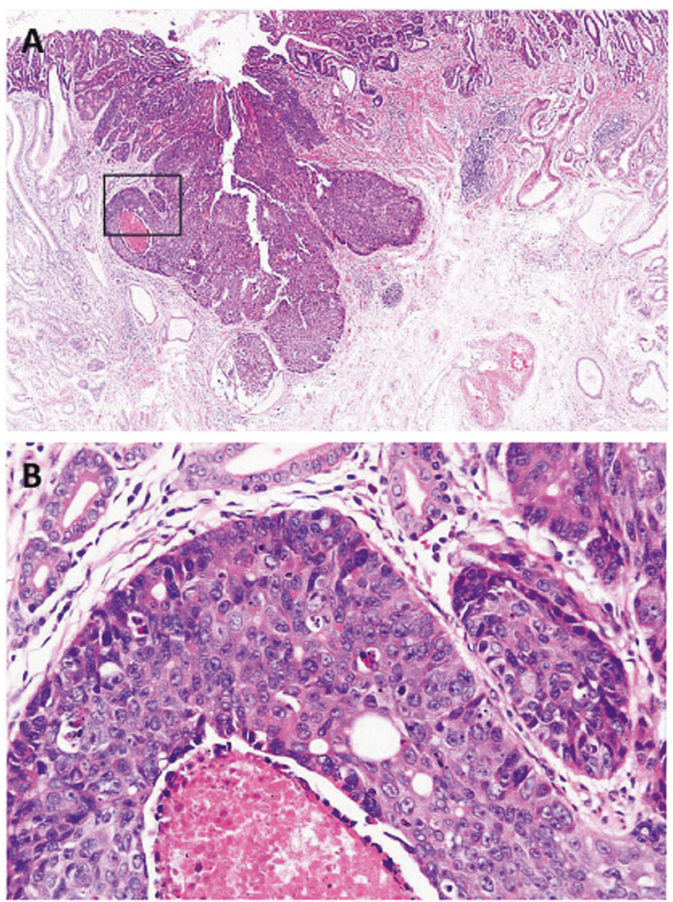

Fig. 3 Early gastric cardiac adenosquamous carcinoma exhibiting a sharp tumor invasion front in the submucosa (a). The squared area in (a) was enlarged in (b) to demonstrate both adenocarcinoma (glands) and squamous cell carcinoma components. Note the comedo necrosis in the squamous cell carcinoma component

frequency of lymph node metastasis was significantly lower for these 33 early gastric cardiac carcinoma cases with 10/115 (8.7\%) for SM1 and 23/187 (12.3\%) for SM2, compared with early gastric non-cardiac carcinomas $(26.1 \%, 213 / 817)$ with $58 / 316(18.4 \%)$ for SM1 $(p<0.05)$ and $155 / 501(30.9 \%)$ for SM2 $(p<0.0001)$, respectively.

\section{Discussion}

In this multicenter study of 495 early gastric cardiac carcinoma cases, we showed a lack of lymph node metastasis in intramucosal carcinoma and uncommon types of carcinomas, such as neuroendocrine and adenosquamous carcinomas, carcinoma with lymphoid stroma, and mixed mucinous and tubular/papillary adenocarcinomas, regardless of the tumor gross growth pattern, size, and invasion depth. In contrast, lymphovascular invasion and poor tumor differentiation in early gastric cardiac carcinomas were unveiled as two significant independent risk factors of lymph node metastasis. On the other hand, the tumor gross ulcerative pattern, tumor size $>3 \mathrm{~cm}$, and submucosal invasion were not identified as significant independent risk factors for lymph node metastasis in early gastric cardiac carcinomas. Overall, our data suggest a very low risk of lymph node metastasis in early gastric cardiac carcinomas, as reported previously in single-center studies [2, 8, 18, 19].
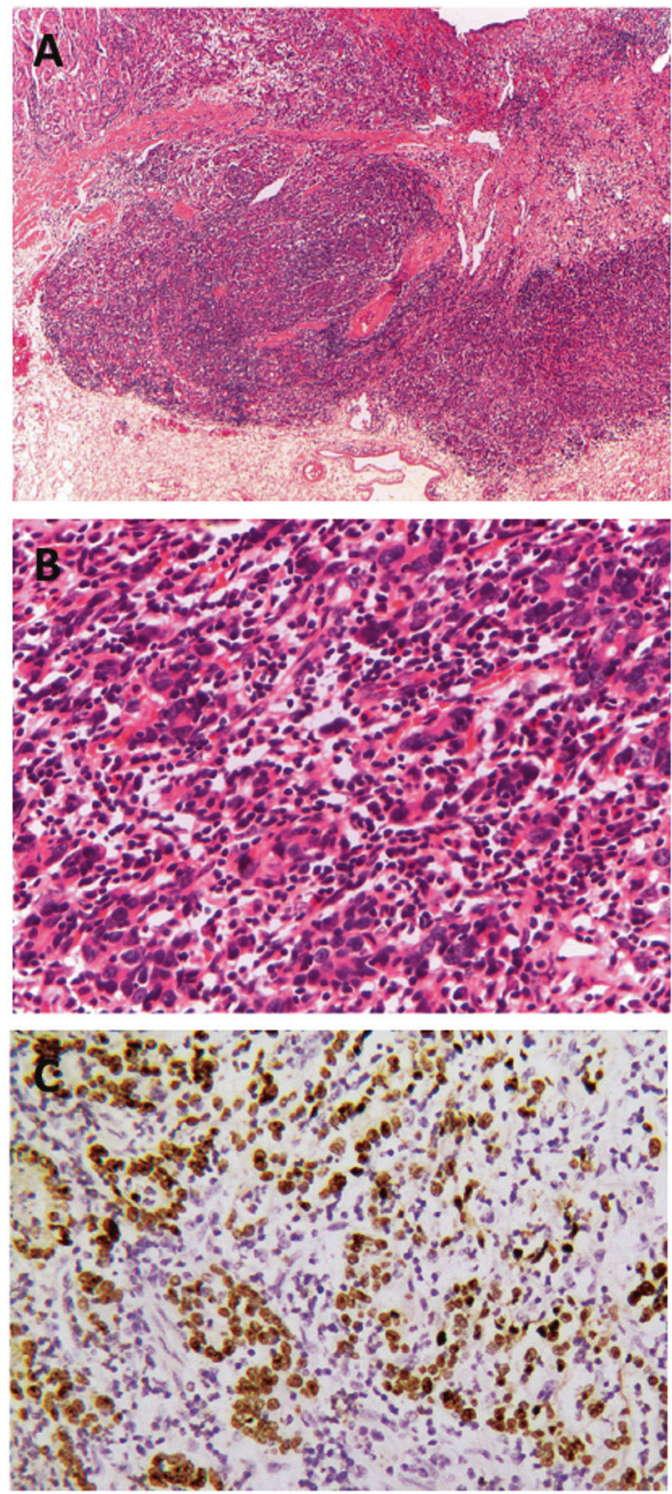

Fig. 4 Early gastric cardiac carcinoma with lymphoid stroma featuring an ulcerated surface, a pushing submucosal invasion border (a), and heavy infiltration of small lymphocytes into poorly differentiated tubular adenocarcinoma (b). Nuclei of poorly differentiated tubular adenocarcinoma were decorated by a positive nuclear in situ hybridization stain for the Epstein-Barr viral antigen (c)

Uncommon histologic types of early gastric carcinomas, such as adenosquamous and neuroendocrine carcinomas as well as carcinoma with lymphoid stroma arise primarily in the gastric cardia and share some common pathologic characteristics, such as a large size, submucosal invasion, and a pushing border. Our findings of the lack of lymph node metastasis in those uncommon types of early gastric carcinomas in the gastric cardia parallel to those of previous reports [2, 20,21], especially for carcinomas with lymphoid stroma that occur mainly in the proximal stomach with Epstein-Barr viral infection and frequent surface ulceration and submucosal invasion $[2,3,21]$. 
Table 2 Independent risk factors of lymph node metastasis in early (pT1) gastric cardiac carcinoma

\begin{tabular}{llll}
\hline Clinicopathologic factor & & $\begin{array}{l}\text { Odds ratio (95\% confidence } \\
\text { interval) }\end{array}$ & $p$ value \\
\hline Histology type & $\begin{array}{l}\text { Tubular adenocarcinoma } \\
\text { Papillary adenocarcinoma } \\
\text { Poorly cohesive carcinoma }\end{array}$ & Reference & \\
& Mucinous carcinoma & & \\
& Micropapillary adenocarcinoma & & NS \\
& Mixed PCC and & & \\
& adenocarcinoma & & \\
& Well & Reference & \\
Differentiation & Moderate & $6.043(1.411-25.879)$ & $<0.05$ \\
& Poorly & Reference & $<0.0001$ \\
Lymphovascular invasion & Absence & $7.6(2.754-20.240)$ & \\
Perineural invasion & Presence & Reference & \\
\hline
\end{tabular}

Table 3 Comparison of relationship of invasion depth and nodal metastasis between early gastric cardiac (EGCC) and non-cardiac (EGNCC) carcinomas

\begin{tabular}{|c|c|c|c|c|}
\hline $\begin{array}{l}\text { Invasion } \\
\text { depth }\end{array}$ & $\begin{array}{l}\text { Total number } \\
(\%)\end{array}$ & $\begin{array}{l}\text { EGCC } \\
(\%)\end{array}$ & EGNCC (\%) & $p$ value \\
\hline \multicolumn{5}{|l|}{ M2 } \\
\hline with LNM & $28(6.2)$ & 0 & $28(7.2)$ & $<0.05$ \\
\hline No LNM & $423(93.8)$ & $63(100.0)$ & $360(92.8)$ & \\
\hline \multicolumn{5}{|l|}{ M3 } \\
\hline with LNM & $34(6.4)$ & 0 & $34(8.5)$ & $<0.0001$ \\
\hline No LNM & 497 (93.6) & $\begin{array}{l}130 \\
(100.0)\end{array}$ & 367 (91.5) & \\
\hline \multicolumn{5}{|l|}{$\mathrm{M} 2$ and M3 } \\
\hline with LNM & $62(6.3)$ & 0 & $62(7.9)$ & $<0.0001$ \\
\hline No LNM & $920(93.7)$ & $\begin{array}{l}193 \\
(100.0)\end{array}$ & $727(92.1)$ & \\
\hline \multicolumn{5}{|l|}{ SM1 } \\
\hline with LNM & $68(15.8)$ & $10(8.7)$ & $58(18.4)$ & $<0.05$ \\
\hline No LNM & $363(84.2)$ & $105(91.3)$ & 258 (81.6) & \\
\hline \multicolumn{5}{|l|}{ SM2 } \\
\hline with LNM & 178 (25.9) & $23(12.3)$ & 155 (30.9) & $<0.0001$ \\
\hline No LNM & $510(74.1)$ & $164(87.7)$ & $346(69.1)$ & \\
\hline \multicolumn{5}{|c|}{$\mathrm{SM} 1$ and SM2 } \\
\hline with LNM & $246(22.0)$ & $33(10.9)$ & $213(26.1)$ & $<0.0001$ \\
\hline No LNM & $873(78.0)$ & $269(89.1)$ & 604 (73.9) & \\
\hline
\end{tabular}

Most gastric cardiac carcinomas correspond to Siewert type II and type III adenocarcinomas of the gastroesophageal junction $[13,22]$. Lymph node metastasis in early gastroesophageal junction carcinomas occurs predominantly in the upper abdomen and very infrequently in the lower mediastinum [23-25]. Matsuda et al. investigated lymph node metastasis in the lower mediastinum in 15 patients with early gastric cardiac carcinoma (cT1) and reported the absence of lymph nodal metastasis in all sentinel lymph nodes identified in the lower mediastinum (5 patients staged as pT1a, 10 as pT1b). No tumor recurrence occurred in all patients without sentinel nodal metastasis during the 38-month follow-up period [26]. Those results, taken along with our findings of the absence of nodal metastasis in the abdominal lymph nodes in intramucosal and rare types of early gastric cardiac carcinomas lend support to the role of endoscopic therapy, especially by endoscopic submucosal dissection, in early gastric cardiac carcinoma resections as a safe alternative to surgical resection of this early cancer $[4,5]$.

In early gastric carcinoma, including early gastric cardiac carcinoma, lymphovascular invasion and poor tumor differentiation have been repeatedly demonstrated as the most important risk factors for lymph node metastasis [11, 18, 19, 27-29] as confirmed in this study. These features have been incorporated into many practice guidelines as contraindications of endoscopic therapy [30-32]. From the pathologist's point of view, these two histology features may be diagnosed with a minimal inter-observer variation. Therefore, in a biopsy specimen from the gastric cardia, it is essential for the pathologists to document tumor histology type, size, differentiation, and the presence or absence of lymphovascular invasion, among other parameters, to guide clinical management of patients with early gastric cardiac carcinoma.

The high risk of lymph node metastasis in poorly cohesive carcinoma is well known [11, 27-29] but not validated in this study on early gastric cardiac carcinoma. The discrepancy may be related to the small sample size $(2.2 \%, 11 /$ 495) for this carcinoma in the current study since poorly cohesive carcinoma arises primarily in the distal stomach 
and rarely in the cardia [2,33]. Although we observed only two cases with lymph node metastasis, poorly cohesive carcinoma mixed with tubular/papillary adenocarcinoma components demonstrated a significantly increased risk of lymph node metastasis [34]. Although additional risk factors such as submucosal invasion certainly contribute to this high risk, the underlying complex multidirectional differentiation in mixed poorly differentiated carcinomas may hold the key for tumor spread and lymph node metastasis. This speculation requires further investigation.

Early gastric cardiac carcinoma has a high propensity for submucosal invasion [2, 8, 26, 33]. In an early study in Japan on early gastric cardiac carcinoma, Tanaka et al. reported the presence of submucosal invasion in $88.9 \%$ (8/ 9) cases [8], which is a bit higher than that $(61 \%, 302 / 495)$ in our cohort. Despite deeper submucosal invasion, lymph node metastasis was absent in all eight cases in that Japanese study and detected in only $33(10.9 \%)$ cases in this cohort, which was a significantly lower proportion than that for early gastric non-cardiac carcinoma $(26.1 \%, 213 / 817)$. Although the reason for the significantly lower risk of lymph node metastasis in submucosal early gastric cardiac carcinoma is unknown, marked fibromuscular hyperplasia in the gastric cardiac region with the frequent presence of "the double muscularis mucosae" is well known and may be one of the plausible defense mechanisms against lymph node metastasis in early gastric cardiac carcinoma [35].

The risk of lymph node metastasis in early gastric carcinoma has been reported not to be gastric region-dependent in many Japanese and Korean studies [11, 27, 36-38], which differs from our findings. This difference appears to result from various disease classification systems used in different countries. In Japan and Korea, gastric cardiac cancer is rare $[39,40]$ and is classified along with upper third gastric cancers [30], which includes cancers in the fundus and proximal corpus as well as in the gastric cardia. From the pathologic point of view, early gastric cardiac carcinoma differs from early gastric non-cardiac carcinoma in the gastric fundus and proximal corpus in multiple ways. First, the cellular origins for early gastric cardiac carcinoma are much more complex and tumors may arise in cardiac, mixed mucoxyntic mucosa, and residual embryonic stem cells, among others [41]. In contrast, the cells of origin for early gastric non-cardiac carcinoma of the fundus and proximal corpus may be related to oxyntic mucosa only. Second, most early gastric cardiac carcinoma tumors are Lauren intestinaltype adenocarcinomas [2, 3, 42], whereas carcinomas in the gastric fundus show considerable proportions of undifferentiated poorly cohesive carcinoma [43]. Third, hyperplastic fibromuscular tissue is present in the gastric cardia but absent from the fundus and corpus. This may explain a lower rate of lymph node metastasis in early gastric cardiac carcinoma compared to early gastric non-cardiac carcinoma, as also reported previously in other studies $[2,8,18,19]$.

There are several strengths of this study: (1) a large number of consecutive early gastric cardiac carcinoma surgical resection cases $(N=495)$ specifically for investigation of risk factors of lymph node metastasis, which is unprecedented; (2) minimal confounding factors in the study patient population, such as Barret's adenocarcinoma, which was intentionally excluded; and (3) uniform execution with a stringent investigation protocol by specialized, experienced gastrointestinal pathologists guided with the World Health Organization diagnostic criteria on early gastric carcinoma. An important limitation of our analysis is not unique to our study but commonly associated with any retrospective multicenter histopathology study on lymph node metastasis in gastric cancer: we could not control for the surgical methods of lymphadenectomy, which is often inconsistent among surgeons, surgical centers, and countries. As such, variation in the number of lymph nodes retrieved was unavoidably present. However, the average number of lymph nodes studied in the cohort was high. We are confident that any analysis on the risk of lymph node metastasis in early gastric cardiac carcinoma would have similar results.

In summary, we demonstrated, in this multicenter study, the absence of lymph node metastasis in intramucosal and uncommon types of early gastric cardiac carcinomas, irrespective of tumor gross patterns, size, and invasion depth. Even in early gastric cardiac carcinomas with submucosal invasion, the risk of lymph node metastasis was still significantly lower than that in early gastric non-cardiac carcinoma, especially for early gastric cardiac carcinoma without lymphovascular invasion and poor tumor differentiation.

Funding The study was partially financed by research grants from the Science and Technology Development Project of the Nanjing City (ZKX05013, ZKX07011 to QH) and a special grant from the Nanjing Drum Tower Hospital in Nanjing, China.

\section{Compliance with ethical standards}

Conflict of interest The authors declare that they have no conflict of interest.

\section{References}

1. Verdecchia A, Corazziari I, Gatta G, et al. Explaining gastric cancer survival differences among European countries. Int J Cancer. 2004;109:737-41.

2. Huang Q, Fang C, Shi J, et al. Differences in clinicopathology of early gastric carcinoma between proximal and distal location in 438 Chinese patients. Sci Rep. 2015;5:13439.

3. Huang Q, Shi J, Sun Q, et al. Clinicopathological characterisation of small ( $2 \mathrm{~cm}$ or less) proximal and distal gastric carcinomas in a Chinese population. Pathology. 2015;47:526-32. 
4. Zhong YS, Yao LQ, Zhou PH, et al. Evaluation of endoscopic submucosal dissection for early tumors located at the esophagogastric junction. Chin J Dig Surg. 2011;10:185-87.

5. Kim JK, Kim GH, Lee BE, et al. Endoscopic submucosal dissection for esophagogastric junction tumors: a single-center experience. Surg Endosc. 2018;32:760-69.

6. Hahn KY, Park CH, Lee YK, et al. Comparative study between endoscopic submucosal dissection and surgery in patients with early gastric cancer. Surg Endosc. 2018;32:73-86.

7. Colquhoun A, Arnold M, Ferlay J, et al. Global patterns of cardia and non-cardia gastric cancer incidence in 2012. Gut. 2015;64:1881-8.

8. Tanaka T, Takeda J, Hashimoto K, et al. Clinical and pathological evaluation of early cancer in the gastric cardia. Kurum Med J. 1990;37:265-69.

9. van Sandick JW, van Lanschot JJ, et al. Pathology of early invasive adenocarcinoma of the esophagus oresophagogastric junction: implications for therapeutic decision making. Cancer. 2000;88:2429-37.

10. Nesi G, Basili G, Girardi LR, et al. Pathological predictors of lymph node involvement in submucosal gastric carcinoma: a retrospective analysis of long-term outcome. In Vivo. 2009;23:337-41.

11. Kang HJ, Kim DH, Jeon TY, et al. Lymph node metastasis from intestinal-type early gastric cancer: experience in a single institution and reassessment of the extendedcriteria for endoscopic submucosal dissection. Gastrointest Endosc. 2010;72:508-55.

12. American Joint Committee on Cancer. Chapter 10. Esophagus and esophagogastric junction. In: AJCC Cancer Staging Manual 7th edn. Springer: New York, NY, 2009, pp 129-44.

13. Huang Q, Fan XS, Agoston AT, et al. Comparison of gastroesophageal Junction carcinomas in Chinese versus american patients. Histopathology. 2011;59:188-97.

14. Huang Q, Fang DC, Yu CG, et al. Barrett's esophagus-related diseases remain uncommon in China. J Dig Dis. 2011;12:420-7.

15. Huang Q, Shi J, Sun Q, et al. Distal esophageal carcinomas in Chinese patients vary widely in histopathology, but adenocarcinomas remain rare. Hum Pathol. 2012;43:2138-48.

16. Huang Q. Definition of the esophagogastric junction: a critical mini review. Arch Pathol Lab Med. 2011;135:384-89.

17. Lauwers GY, Carneiro F, Graham DY, et al.. Gastric carcinoma. In: Bosman FT, Carneiro F, Hruban RH, Theise ND, (eds). WHO Classification of Tumours of the Digestive System. Lyon, France: IARC Press; 2010. pp 48-58.

18. Zhao BW, Chen YM, Jiang SS, et al. Lymph node metastasis, a unique independent prognostic factor in1 early gastric cancer. PLoS ONE. 2015;10:e0129531.

19. Fang C, Shi J, Sun Q, et al. Risk factors of lymph node metastasis in early gastric carcinomas diagnosed by WHO criteria in 379 Chinese patients. J Dig Dis. 2016;17:526-37.

20. Huh CW, Jung DH, Kim H, et al. Clinicopathologic features of gastric carcinoma with lymphoid stroma in early gastric cancer. $\mathbf{J}$ Surg Oncol. 2016;114:769-72.

21. Lim H,Lee IS,Lee JH, et al. Clinical application of early gastric carcinoma with lymphoid stroma based on lymph node metastasis status. Gastric Cancer. 2017;20:793-801.

22. Siewert JR, Feith M, Stein HJ. Biologic and clinical variations of adenocarcinoma at the esophago-gastric junction: relevance of a topographic-anatomic subclassification. J Surg Oncol. 2005;90:139-46.

23. Kakeji Y, Yamamoto M, Ito S, et al. Lymph node metastasis from cancer of the esophagogastric junction, and determination of the appropriate nodal dissection. Surg Today. 2012;42:351-58.
24. Okholm C, Svendsen LB, Achiam MP. Status and prognosis of lymph node metastasis in patients with cardia cancer - a systematic review. Surg Oncol. 2014;23:140-46.

25. Yamashita H, Seto $Y$, Sano T, et al. Results of a nation-wide retrospective study of lymphadenectomy for esophagogastric junction carcinoma. Gastric Cancer. 2017;20:69-83.

26. Matsuda T, Takeuchi H, Tsuwano S, et al. Sentinel node mapping in adenocarcinoma of the esophagogastric junction. World J Surg. 2014;38:2337-44.

27. Gotoda T, Yanagisawa A, Sasako M, et al. Incidence of lymph node metastasis from early gastric cancer: estimation with a large number of cases at two large centers. Gastric Cancer. 2000;3:219-25.

28. Zhao X, Cai A, Xi H, et al. Predictive factors for lymph node metastasis in undifferentiated early gastric cancer: a systematic review and meta-analysis. J Gastrointest Surg. 2017;21:700-11.

29. Ronellenfitsch U, Lippert C, Grobholz R, et al. Histology-based prediction of lymph node metastases in early gastric cancer as decision guidance for endoscopic resection. Oncotarget. 2016;7:10676-83.

30. Japanese Gastric Cancer Association. Japanese gastric cancer treatment guidelines 2014 (ver. 4). Gastric Cancer. 2017;20:1-19.

31. Chinese Society of Digestive Endoscopy Consensus for early gastric carcinomadiagnosis and treatment in China. Chin J Dig Endosc. 2014;31:361-77.

32. Ajani JA, D'amico TA, Almhanna K, et al. Esophageal and esophagogastric junction cancers, version 1.2015[J]. J Natl Compr Canc Netw. 2015;13:194-227.

33. Okabayashi T, Gotoda T, Kondo H, et al. Early carcinoma of the gastric cardia in Japan: is it different from that in the West? Cancer. 2000;89:2555-9.

34. Hanaoka N, Tanabe S, Mikami T, et al. Mixed-histologic-type submucosal invasive gastric cancer as a risk factor for lymph node metastasis: feasibility of endoscopic submucosal dissection. Endoscopy. 2009;41:427-32.

35. Aida J, Vieth M, Ell C, et al. Palisade vessels as a new histologic marker of esophageal origin in ER specimens from columnarlined esophagus. Am J Surg Pathol. 2011;35:1140-5.

36. Nam MJ, Oh SJ, Oh CA, et al. Frequency and predictive factors of lymph node metastasis in mucosal cancer. J Gastric Cancer. 2010;10:162-7.

37. Lim MS, Lee HW, Im H, et al. Predictable factors for lymph node metastasis in early gastric cancer-analysis of single institutional experience. J Gastrointest Surg. 2011;15:1783-8.

38. Sekiguchi M, Oda I, Taniguchi $\mathrm{H}$, et al. Risk stratification and predictive risk-scoring model for lymph node metastasis in early gastric cancer. J Gastroenterol. 2016;51:961-70.

39. Maeda H, Okabayashi T, Nishimori I, et al. Clinicopathologic features of adenocarcinoma at the gastric cardia: is it different from distal cancer of the stomach? J Am Coll Surg. 2008;206:306-10.

40. Sano T, Coit DG, Kim HH, et al. Proposal of a new stage grouping of gastric cancer for TNM classification: International Gastric Cancer Association staging project. Gastric Cancer. 2017;20:217-25.

41. Huang Q, Sun Q, Fan XS, et al. Recent advances in proximal gastric carcinoma. J Dig Dis. 2016;17:421-32.

42. Tajima Y, Nakanishi Y, Yoshino T, et al. Clinicopathological study of early adenocarcinoma of the gastric cardia: comparison with early adenocarcinoma of the distal stomach and esophagus. Oncology. 2001;61:1-9.

43. Kumagai K, Maekawa K, Urabe M, et al. Clinicopathological study of early gastric carcinoma of the cardiac portion. Gan No Rinsho. 1985;31:31-36. 